\title{
Joint Simulation of Trolley Vehicle-Frame Structure Coupled Vibration Using ADAMS and ANSYS for Container Crane Simulated Training System
}

\author{
Kai-liang Lu*, You-fang Huang, Wei Yan, Jun Hu, Chao Chen and Dao-fang Chang \\ Logistics Engineering College, Shanghai Maritime University \\ lkl1984@163.com
}

\begin{abstract}
Some progress has been made in container crane simulated training system research and development globally, but there are still serious problems such as faultiness in training function and effect, less coordination in synchronicity of visual and tactile simulation, etc. The lack of accurate system dynamics model is the main reason. The existing model takes the consideration of real-time motion simulation so simplified that it's unable to accurately reflect the dynamic characteristics of structure or mechanism in starting and braking stage, and the coupled vibration characteristics in operation process, etc. In this paper, the geometric model of the entire container crane structure was established by using Solidworks, and used ADAMS and ANSYS for joint simulation of start-brake and coupled vibration characteristics, which provides a solution to establishing kernel model of container crane simulated training system and achieving real-time simulation.
\end{abstract}

Keywords: Container Crane; Simulated Training System; Dynamics Simulation; Coupled Vibration; ADAMS; ANSYS

\section{Introduction to container crane simulated training system}

Simulated training system (referred to as the simulator) is a multidisciplinary virtual reality (VR) system [1-3], which consists of technology of system dynamics simulation, real-time 3D visual simulation, sensor and control technology, computer interface and communication, artificial intelligence and knowledge engineering, and multimedia and network. Compared with the real machine training, the simulator is prominently dominant in areas of safety, economical efficiency, area of application, training effect, and is unstinted in applicable training places. With vigorous development in recent years, there are various simulators of aircraft, ship, car, train/subway, port machineries like container crane, and other engineering machinery and vehicles, etc. [3].

In the aspect of crane simulator, since the 1980s onwards, many companies had carried out relevant research work, like Dutch BOS/CAT, American Globalsim and MPRI Ship Analytics, Norwegian Hitec-O, British TSI, Korean TSB, Canadian Simlog [4]. Among them, GlobalSim's MasterLift ${ }^{\mathrm{TM}}$ series ML4000 type crane simulator (shown in Figure 1) includes hardware components: multiple-crane-integrated controlling platform, perfect functional teacher console, multiple degrees of freedom (MDOF) vibration platform, hemisphere visual integrated screen projection system, and realistic audio system. Its advantages lie in: real effect of visual simulation, a strong sense of immersion, a kinematics model established to simulate the object, implementation of real-time motion synchronous simulation in visual, 
tactile and auditory sense. The disadvantage is the vibration platform cannot simulate simultaneously the vibration of dynamic seat with the visual simulation.

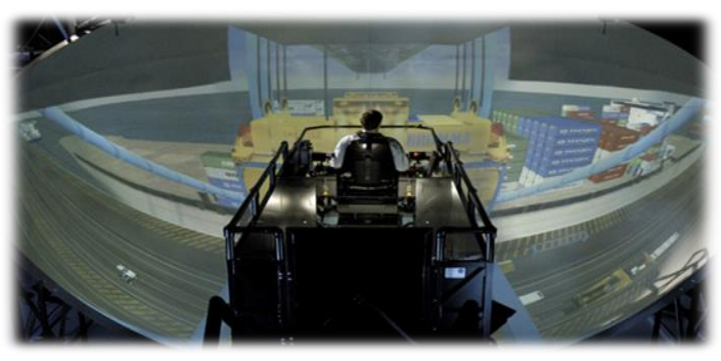

(a) System panorama

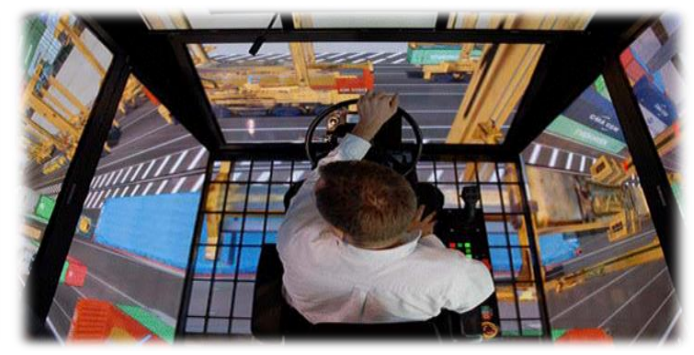

(b) Visual simulation screen

Figure 1. GlobalSim Company's ML4000 type crane simulator of MasterLift ${ }^{\mathrm{TM}}$ series

Many domestic research institutes had begun to research and develop crane simulator from late 1990s, such as Shanghai Maritime University, Wuhan University of Technology, Water carriage research institute of Ministry of Communications, etc. [1-5]. The current status and characteristics of technology can be summarized as follows. In the aspect of simulation model and its solution methods, the vehicle - pendulum dynamic model is widely adopted, which is based on the principle of theoretical mechanics; According to the mechanical transmission theory, list transmission equation of gantry travel, trolley travel and hoisting mechanism; Using explicit numerical integration algorithm combined with predictor-corrector formula to calculate the dynamic response. In the aspect of visual simulation, the real-time visual model is mainly established by MultiGen Creator software, and Vega or OpenGVS for graphics driving according to the dynamic response data. In the aspect of tactile simulation, a beneficial attempt has been done reported in the literature [5], where the control scheme of servo motor, electric cylinder and other composed half closed loops was adopted to design the driver seat single degree of freedom (SDOF) vibration system which can simulate the gantry or trolley travelling vibration. In the aspect of auditory simulation, basically it is recorded on the spot and then playback with the scene. The simulator system structure diagram is shown in Figure 2.

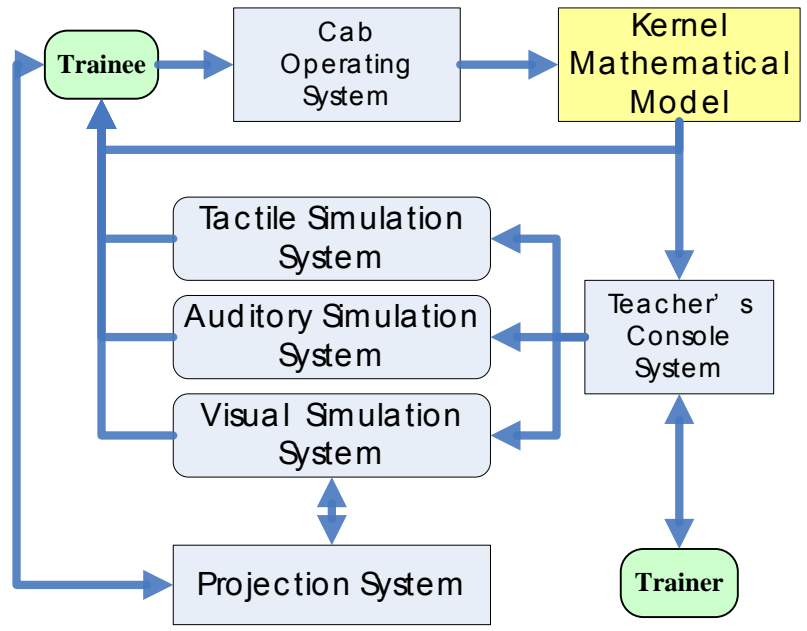

Figure 2. The simulator system structure diagram 
Therefore, some progress has been made in container crane simulated training system research and development at home and abroad, especially its visual simulation effect, fidelity of simulation environment has been able to produce good sense of immersion. But there are still prominent problems, such as imperfect training function and effect, discordant synchronization of visual and tactile simulation, mainly exposed in: (1) The image motion in visual simulation based on the laws of physics is too simple, and many key operations like trolley control and spreader on the container cannot be implemented correctly, which is extremely important to driver training. (2) Driving experience feeling (the sense of motion and vibration) failed to be fully experienced, and it is inharmonious with visual simulation motion.

The main reason is analyzed to be the lack of accurate system dynamics model. The existing model, considering of real-time dynamic simulation, is overly simplified. The system degree of freedoms are too small, and the structural deformation and vibration as well as interaction between mechanisms is ignored. Therefore, the simplified model cannot accurately reflect start and brake dynamics characteristics of structure or mechanism, coupled vibration characteristics in operation process, torsional pendulum characteristics of the slingsteel-wire-rope system, and so on. It causes the real-time driver's seat vibration simulation system to fail to response immediately, and it need to collect vibration signals in different conditions on the spot, so it's difficult to ensure synchronization with visual motion.

\section{Research Review of Dynamics Simulation for Container Crane}

Container crane dynamics simulation is mainly about simulating the structure or mechanism's dynamic characteristics in the working conditions of gantry travel, trolley travel and hoisting. The accurate system dynamics model is the core content; improving the computational efficiency on the premise of the simulation accuracy is the key problem. Computational multi-body system dynamics is an effective way to solve this problem, general and special software are emerging constantly, like ADAMS, DADS, SIMPACK, WorkingModel, Recurdyn, CADAMB and so on [6, 7]. Although the general software can achieve real-time simulation, it can only achieve the data result interaction and it's not easy to do real-time interactive simulation. Therefore, establishing a systemic multi-body dynamics model as the kernel algorithm or developing unique software which can do real-time interaction with visual simulation software is the feasible scheme and key problem of the container crane simulator real-time dynamic simulation. Taichi [8] established the 6DOF trolley travel multi-body dynamics model for driving simulator and achieved the prediction of dynamic system performance and comfort evaluation. The real time solution results were calculated by the approximate analysis method, in the process of trolley travel simulation with the 91dof system. In this case, the real time solution can be achieved by using $2 \mathrm{~ms}$ as numerical integration time step. Multi-body dynamics model like the simplified model [4, 5], relative to the real system or finite element model, can greatly reduce the degree of freedom of the system and improve the computational efficiency.

Simulating structural coupled vibration characteristics under gantry travel and trolley travel's working conditions is the key issue in real-time dynamic model for the container crane simulator. At present, research on structural coupled vibration problem in container crane and other port machinery has seldom been reported. Lu K.L. [9] had a preliminary discussion to this question, based on the free-interface modal synthesis method, the coupled vibration time-domain responses of a container vehicle-truss bridge system used for transporting containers between the port and the yard in automated container terminal, 
inspired by self-excitation including track irregularity and hunting movement as well as wind and seismic load, were obtained, with the methods of theoretical calculation, numerical simulation and miniature model test.

Vehicle and bridge coupled vibration research has been largely carried out in the area of rail transportation [10-12]. At present, multi-rigid body-spring-damper discrete model as the vehicle model while FEM model as bridge model are commonly used. Then the system equation is formed through wheel-rail interaction. Wheel-rail interaction is usually treated in the following three ways: (1) Both measured track irregularity and hunting movement are assumed to be the wheel-rail interaction [10]. (2) Measured or artificial profile of bogie frame hunting movement is considered as the system input [11]. Main parameters of these two ways come from the measured data which can reflect the main character of relationship between wheel and rail. (3) The creep force and kinematical relation of the wheel-rail model can be calculated iteratively by rolling contact theory, such as Hertz theory [12]. Among the three ways, only the last one is able to determine the contact point and contact force of wheel and rail precisely. However, this procedure is very complicated.

If the aim is not the wheel-rail kinematical relation but the coupled vibration response, for real-time simulation, the first two methods are reasonably effective. By means of modal synthesis method we can form the system equation. The equation is usually solved in the time domain due to time-varying.

In summary, establishing an accurate container crane real-time dynamic model and working out a high efficient algorithm with a proper computational accuracy, realizing the visual, tactile, auditory simulation synchronization are all key problems to further improve the systemic sense of immersion and reality for container crane simulated training system. In this paper, firstly, the Solidworks geometric model of container crane trolley travel and frame structure was built. Then, by using ADAMS and ANSYS, joint simulation of start-brake and coupled vibration characteristics of the container crane under gantry and trolley travelling conditions were studied, which provides a solution to establishing real-time kernel model of container crane simulated training system.

\section{Container Crane Performance Parameters and Geometric Model Building}

\subsection{Frame structure}

Taking a certain type of double 40 feet container crane as the analysis object, the container crane structure's basic performance parameters as listed in Table 1.

Table 1. Basic performance parameters of the container crane

\begin{tabular}{|c|c|c|c|c|c|c|c|}
\hline \multicolumn{2}{|c|}{ Item } & \multirow{2}{*}{\multicolumn{2}{|c|}{$\begin{array}{c}\text { Parameter } \\
45 \mathrm{~T}\end{array}$}} & \multicolumn{2}{|c|}{ Item } & \multicolumn{2}{|c|}{ Parameter } \\
\hline $\begin{array}{l}\text { Rated load } \\
\text { weight }\end{array}$ & \multirow{3}{*}{$\begin{array}{l}\begin{array}{l}\text { Under } \\
\text { spreader }\end{array} \\
\text { Main hoist }\end{array}$} & & & \multirow{2}{*}{ Motor } & \multirow{2}{*}{$\begin{array}{l}\begin{array}{l}\text { Gantry } \\
\text { travel }\end{array} \\
\text { Boom } \\
\text { pitching }\end{array}$} & \multicolumn{2}{|c|}{$16 \times 11 \mathrm{KW} 1750 \mathrm{rpm}$} \\
\hline \multirow{5}{*}{ Speed } & & $40 \mathrm{~T}$ load & $60 \mathrm{~m} / \mathrm{min}$ & & & \multicolumn{2}{|c|}{$1 \times 95 \mathrm{KW} \quad 1750 \mathrm{rpm}$} \\
\hline & & Zero load & $120 \mathrm{~m} / \mathrm{min}$ & \multicolumn{2}{|c|}{ Gauge } & \multicolumn{2}{|c|}{$26 \mathrm{~m}$} \\
\hline & Trolley travel & \multicolumn{2}{|c|}{$150 \mathrm{~m} / \mathrm{min}$} & \multicolumn{2}{|c|}{ Front/Rear extension } & \multicolumn{2}{|c|}{$36 \mathrm{~m} / 12 \mathrm{~m}$} \\
\hline & Gantry travel & \multicolumn{2}{|c|}{$45 \mathrm{~m} / \mathrm{min}$} & \multirow{2}{*}{$\begin{array}{l}\text { Lifting } \\
\text { altitude }\end{array}$} & $\begin{array}{l}\text { Above } \\
\text { track }\end{array}$ & \multicolumn{2}{|c|}{$27 \mathrm{~m}$} \\
\hline & $\begin{array}{l}\text { Boom } \\
\text { hoisting time }\end{array}$ & \multicolumn{2}{|c|}{$5 \mathrm{~min}$} & & $\begin{array}{l}\text { Total } \\
\text { height }\end{array}$ & \multicolumn{2}{|c|}{$42 \mathrm{~m}$} \\
\hline \multirow{2}{*}{ Motor } & Main hoist & $2 \times 260 \mathrm{~K}$ & $\begin{array}{l}900 / 1800 \\
n\end{array}$ & \multirow{2}{*}{$\begin{array}{l}\text { Power } \\
\text { source }\end{array}$} & \multirow{2}{*}{ Generator } & $\begin{array}{l}\text { Main } \\
\text { generator }\end{array}$ & $1625 \mathrm{KW}$ \\
\hline & Trolley travel & $4 \times 25 \mathrm{~K}$ & $1750 \mathrm{rpm}$ & & & $\begin{array}{l}\text { Vice } \\
\text { generator }\end{array}$ & $256 \mathrm{KW}$ \\
\hline
\end{tabular}


Solidworks is currently one of the most widely used three-dimensional modeling software. With the advantages of simple operation, rich functions, it can perform 3D modeling accurately, reduce error in the design process and improve the quality of the products. The geometric model of container crane frame structure was built by using Solidworks. Main structure materials are $16 \mathrm{Mn}$ and Q235, by proper simplification, the geometric assembly model of container crane structure is shown in Figure 3.
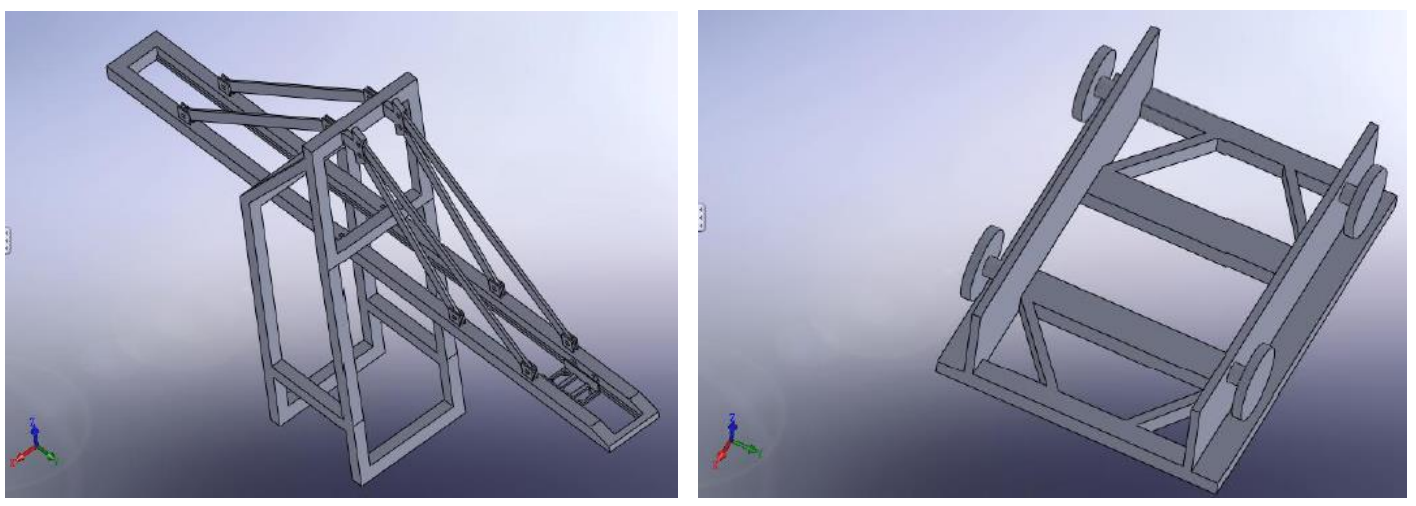

Figure 3. Frame structure geometric model Figure 4. Trolley structure geometric model

\subsection{Trolley travel structure}

The basic performance parameters of trolley travel as listed in Table 2. Similarly, by proper simplification, the trolley travel structure model finished by Solidworks is shown in Figure 4.

Table 2. Basic performance parameters of trolley travel

\begin{tabular}{c|c|l|l|c}
\hline Item & Parameter & \multicolumn{2}{|c|}{ Item } & Parameter \\
\hline Track Type & A75 & \multirow{2}{*}{ Brake } & Type & SB8.11-355X20-30/5 \\
\cline { 4 - 5 } Speed & $150 \mathrm{~m} / \mathrm{min}$ & & Torque & $270 \mathrm{~N} \cdot \mathrm{m}(\mathrm{rated}) 243 \mathrm{~N} \cdot \mathrm{m}(\mathrm{setting})$ \\
\hline Wheel diameter & $630 \mathrm{~mm}$ & \multirow{2}{*}{ Coupling } & Type & MLPK6-355X20-J445A \\
\cline { 4 - 5 } & & & Torque & $1800 \mathrm{~N} \cdot \mathrm{m}$ \\
\hline Motor & $4 \times 25 \mathrm{KW} 1750 \mathrm{rpm}$ & &
\end{tabular}

\section{ADAMS and ANSYS Joint Simulation}

\subsection{Generating flexible body finite element model}

The crane frame and gantry travel structure Solidworks geometric models were exported as .iges format file, and then imported into ANSYS for material attribution giving and meshing. After that, the finite element models of flexible body were generated. Take the crane frame structure for instance, nodes of 5570, 5945, 5876 are selected as the marker points, then the model is exported as .x_t file and finally imported into the ADAMS software. The flexible body ANSYS and ADAMS finite element models (FEM) are respectively shown in Figure 5 and Figure 6.

\subsection{Adding constraint}

After importing the model into ADAMS, the relative motion relationship between two separate parts can be defined by the constraints in the software, so that every component can 
be connected as an entirety. The crane ADAMS model consists of 5 parts, including ground, door frame, the front boom structure, front and rear rods, trolley frame. Add a constraint between each two components, which makes the model an entirety. The constraints are as follows: (1) Add 4 rotation pairs to the hinge of door frame and front boom structure; (2) Add 2 rotation pairs to the hinge of door frame and rear boom structure; (3) Add 6 rotation pairs to the hinge of door frame and rods; (4) Add 1 translation pair to the track of trolley frame and front boom structure; (5) Add 1 translation pair to door frame and the ground.

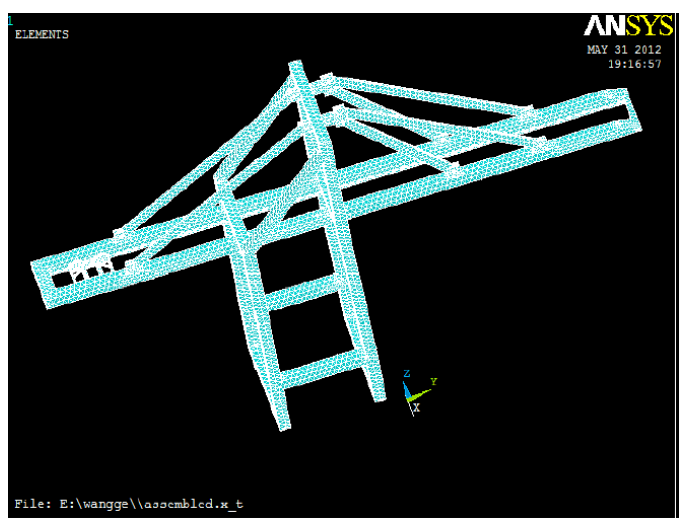

Figure 5. Frame structure ANSYS FEM

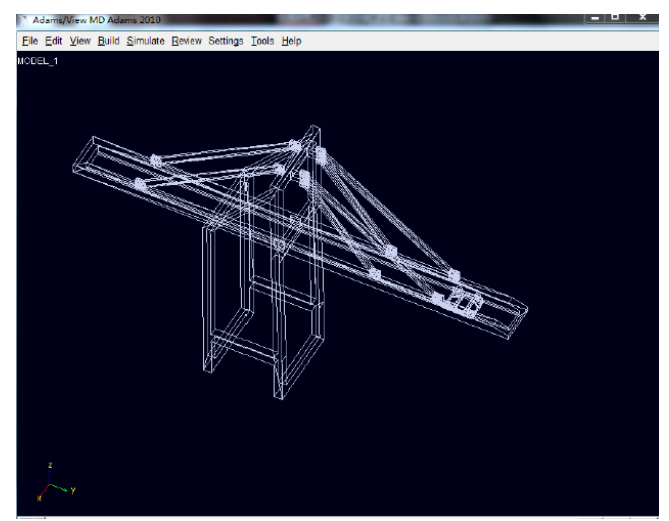

Figure 6. Frame structure ADMAS FEM

\subsection{Defining drive}

In ADAMS, the drive means that the movement of a component is the function of time. In this model, altogether we need to simulate the movements of three mechanisms and add two drives. The drive of gantry travel is added to the translational joint between the door frame structure and the ground. The drive of trolley travel is added to the translational joint between the trolley frame and the track on the boom. The driving function employed is the step function. To complete a working cycle, it needs hoisting, gantry travel and trolley travel mechanisms working together. And every mechanism runs according to the speed in Table 1 and Table 2. Besides, Table 3 lists the working sequence in the whole simulation process and the expression of the STEP driving function.

Table 3. Working sequence and driving function

\begin{tabular}{c|c|c}
\hline Action & Time & Driving function \\
\hline $\begin{array}{c}\text { Gantry travel } \\
\text { goes forward }\end{array}$ & $0 \sim 25 \mathrm{~s}$ & STEP(time, 0, 0, 10, 750)+ STEP(time, 15, 0, 25, -750) \\
\hline $\begin{array}{c}\text { Trolley travel } \\
\text { goes forward }\end{array}$ & $30 \sim 55 \mathrm{~s}$ & STEP(time, 30, 0, 40, 2500)+ STEP(time, 45, 0, 55, -2500) \\
\hline $\begin{array}{c}\text { Trolley travel } \\
\text { goes backward }\end{array}$ & $60 \sim 85 \mathrm{~s}$ & STEP(time, 60, 0, 70, 2500)+ STEP(time, 75, 0, 85, -2500) \\
\hline $\begin{array}{c}\text { Gantry travel } \\
\text { goes backward }\end{array}$ & $90 \sim 115 \mathrm{~s}$ & STEP(time, 90, 0, 100, -750)+ STEP(time, 105, 0, 115, 750) \\
\hline
\end{tabular}

The total time of simulation is 120 seconds. The working condition is the continual process of gantry travel and trolley travel go forward and backward in turns. The driving function of the gantry travel mechanism is: STEP (time, 0, 0, 10, 750) + STEP (time, 15, 0, 25, -750) + STEP (time, 90, 0, 100, 750) + STEP (time, 105, 0, 115, -750). The variable of this function is speed. The gantry travel's speed is $v_{1}=45 \mathrm{~m} / \mathrm{min}=750 \mathrm{~mm} / \mathrm{s}$. Both the starting time and the 
braking time are 10 seconds. The driving function of the trolley travel mechanism is STEP (time, 30, 0, 40, 2500) + STEP (time, 45, 0, 55, -2500) + STEP (time, 60, 0, 70, 2500) + STEP (time, 75, 0, 85, -2500). Its speed is $v_{2}=150 \mathrm{~m} / \mathrm{min}=2500 \mathrm{~mm} / \mathrm{s}$. Both the starting time and the braking time are also 10 seconds. The definitions of gantry travel's and trolley travel's driving functions are shown in Figure 7.

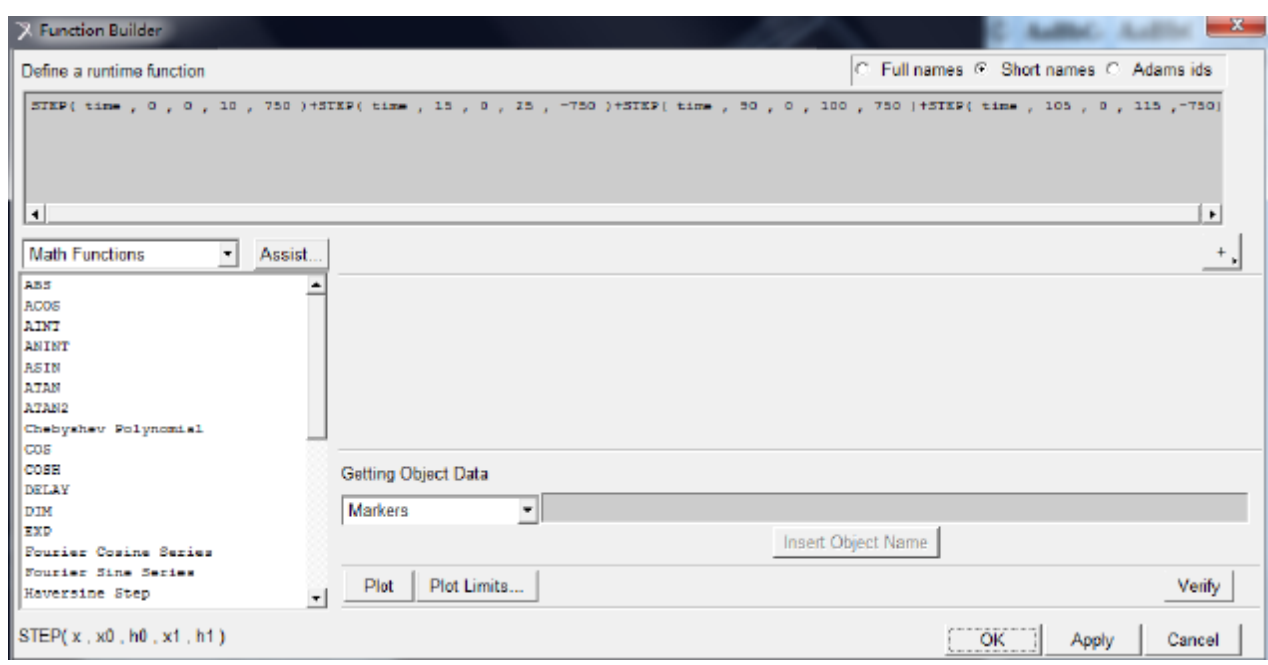

(a) Gantry travel

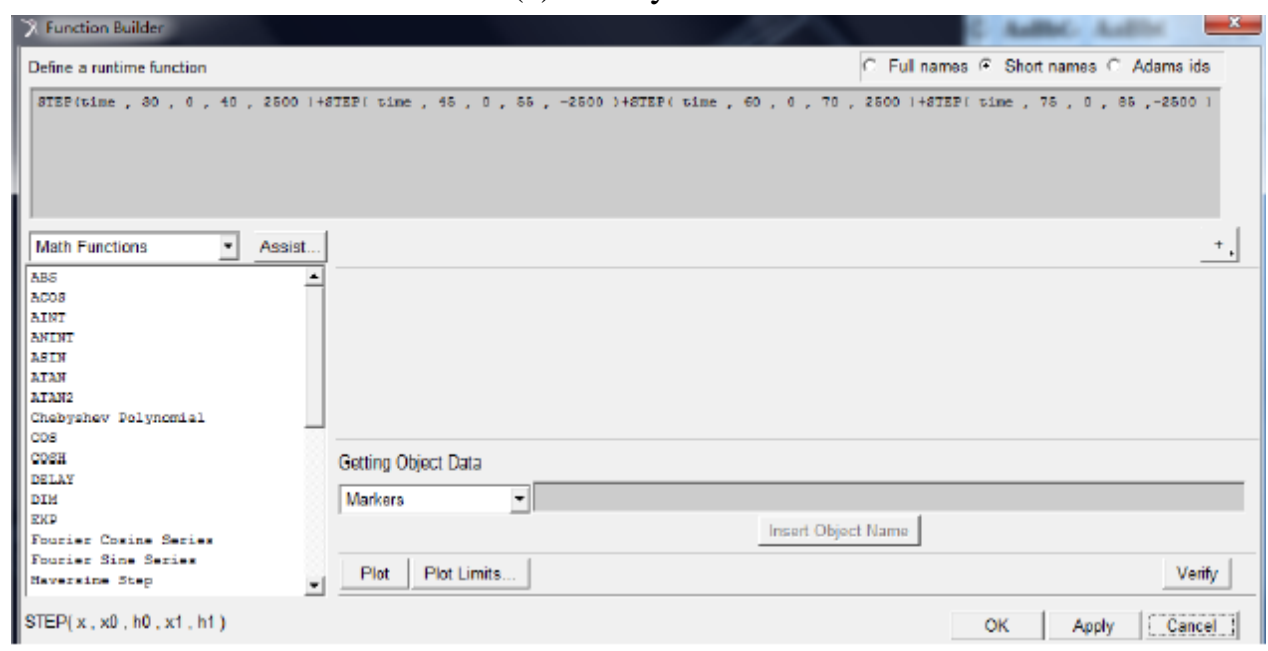

(b) Trolley travel

Figure 7. The definition of gantry travel's and trolley travel's driving functions

\subsection{Coupled vibration response result}

In the working condition of gantry travel, the entire crane frame structure experiences the processes of acceleration, uniform and deceleration. The gantry travel speed time-history curve is shown in Figure 8(a). It can be seen that: Between 0 10s, the frame structure is in accelerated motion, the velocity comes to maximum and then in uniform motion; Between $15 \sim 25 \mathrm{~s}$, the frame structure begins to decelerate; Between 90 115s, repeat the above motion again. Thus, the start or braking movement under typical working conditions during gantry 
travelling can be simulated. The acceleration time-history curve of gantry travel is shown in Figure 8(b).

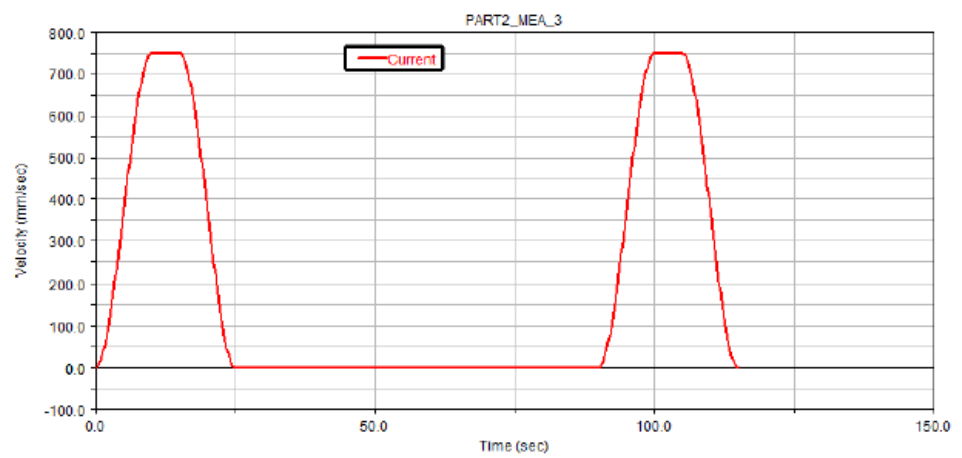

(a) Velocity time-history curve

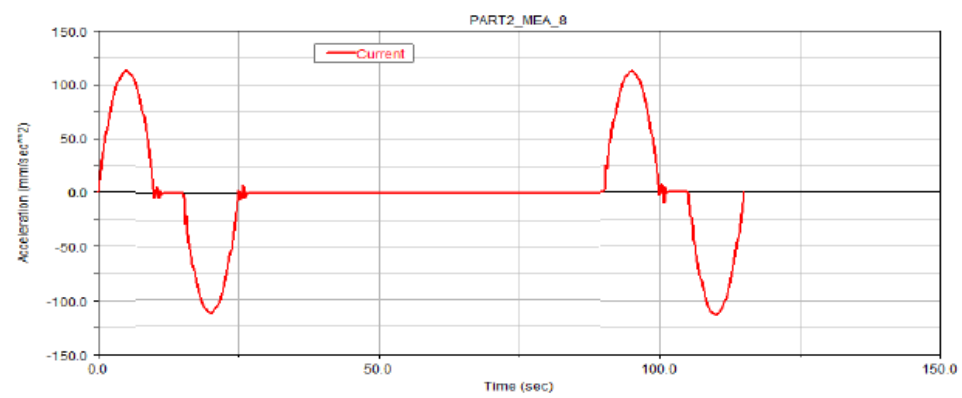

(b) Acceleration time-history curve

\section{Figure 8. Velocity and acceleration time-history curve of gantry travel}

The trolley travel speed time-history curve is shown in Figure 9(a). It can be seen that: the trolley travel frame structure is in accelerated motion between 30 40s till maximum speed is reached, then the uniform motion; the trolley travel speed slows gradually to zero between $45 \sim 55 \mathrm{~s}$ and between $60 \sim 85 \mathrm{~s}$ the trolley travel repeats the above motion again. Thus, the start or braking movement under typical working conditions during trolley travelling can be simulated. The acceleration curve of trolley travel is shown in Figure 9(b).

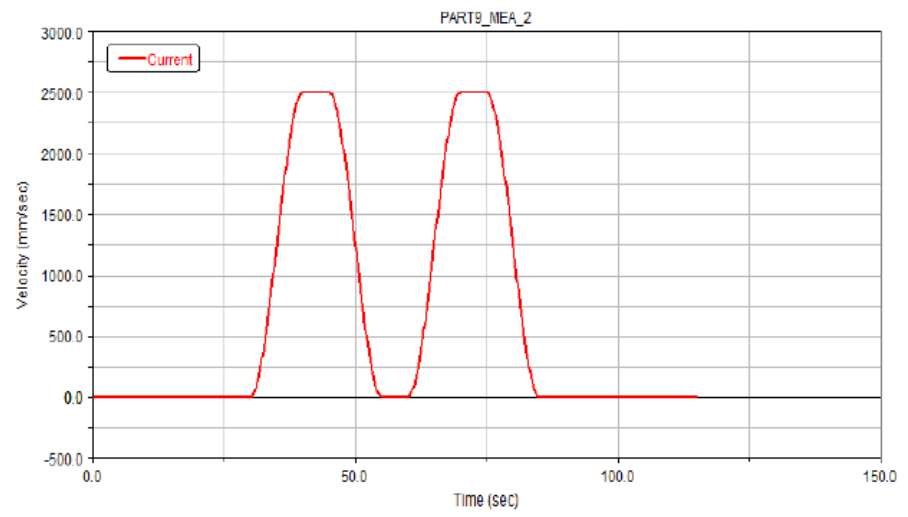

(a) Velocity time-history curve 


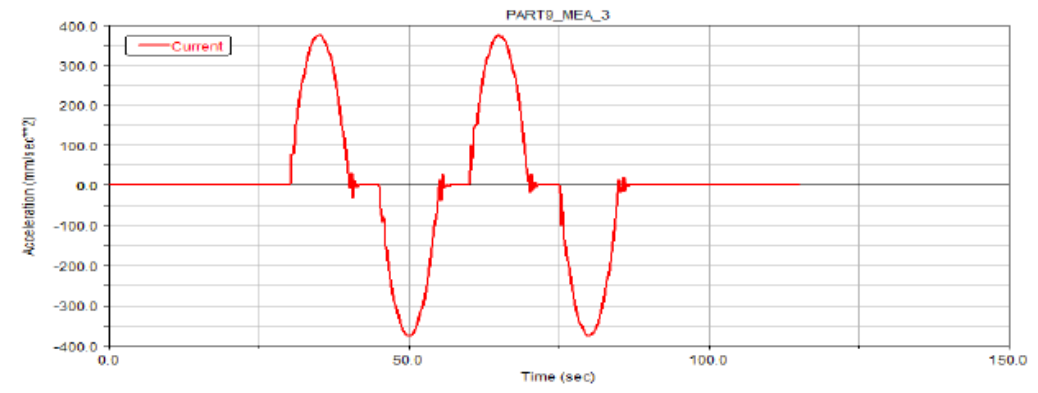

(b) Acceleration time-history curve

Figure 9. Velocity and acceleration time-history curve of trolley travel

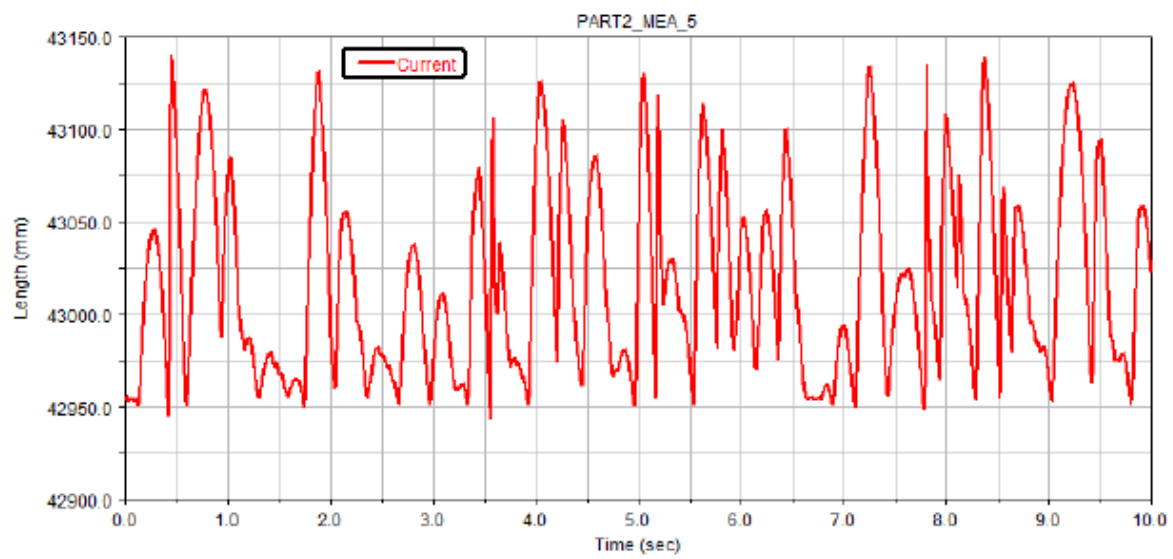

Figure 10. Coupled vibration displacement response time-history curve of boom node

Coupled vibration displacement response time-history curve of the marker point generated from node 5876 on the rear boom is shown in Figure 10. The boom node's response curve is not consistent with the velocity and acceleration time-history curves of the gantry or trolley travel, but showing a certain degree of randomness. This is indicative of the presence of coupling vibration between trolley vehicle and frame structure.

It is known that, as time goes by, the trolley and boom interacts with each other by contact displacement and force. On one hand, the movement of trolley travel causes the deformation of container crane structure by contact force, container crane structure deformation in turn affects the movement of trolley travel which forms interaction; on the other hand, the trolley travel and container crane structure must satisfy compatibility conditions of force and displacement at the point of contact; then the coupled vibration is produced.

Moreover, the computing time is short, on Dell Precision 4600 workstation (Basic configuration: Intel (R) Core(TM) i5-2520M CPU @2.50GHz, 2.49GHz; 3.23G Memory), it takes about $4 \mathrm{~s}$ to finish one case entire calculation (totally 6000 steps). Thus, it can be concluded that by using Solidworks to create geometric model and the joint simulation of ADAMS and ANSYS, the real-time analysis of trolley vehicle - frame structure coupled vibration can be achieved. 


\section{Conclusions and Future Work}

A certain type of double 40 feet container crane was taken as the analysis object. The main structure geometric models of container crane frame structure and trolley travel were established by using Solidworks. Then geometric models were imported into ANSYS to generate flexible body finite element models, combined with ADAMS to create the constraints and define drive. Thus, the container crane dynamics simulation model which can reflect the structural (mechanism) starting and braking dynamics characteristics and the coupling vibration characteristics in the working conditions is formed. The container crane coupling vibration displacement response in the typical working conditions of gantry travelling and trolley travelling was solved. Although the model has been appropriately simplified, the coupling vibration simulation without considering the track irregularity and other factors, the simulation result shows that the method of combining ANSYS with ADAMS to do container crane dynamics simulation providing a feasible solution to establishing kernel mathematic model for container crane simulated training system.

Future research work includes: establishing container crane's structure and mechanism module parametric model, system excitation data acquisition in real machine, study on the effects and sensitivity of various parameters and excitations on the system dynamics, furthermore, building kernel mathematical models and control algorithms for the simulated training system. Then, real-time simulation can be obtained and the visual and tactile simulation synchronization can be improved.

\section{Acknowledgements}

This work is sponsored by Shanghai Top Academic Discipline Project- Management Science \& Engineering, this paper is supported by Doctoral Fund of the Ministry of Education Jointly Funded Project(20123121120002) and Shanghai Education Committee Project "Shanghai Young College Teacher Training Subsidy Scheme", also supported in part by National Natural Science Foundation project(71101090), Ministry of Transport Research Projects (2012-329-810-180), Shanghai Municipal Education Commission Project (12ZZ148, 13YZ080).

\section{References}

[1] B. H. Wilson, R. R. Mourant, M. Li, et al., "A virtual environment for training overhead crane operators: realtime implementation", IIE Transactions, vol. 30, no. 4, (1998), pp. 589-595.

[2] H. Dong, G. Xu and D. Chen, "Research on overhead crane training system and its construction based on virtual reality", Proceedings - ICAIE 2010: International Conference on Artificial Intelligence and Education, (2010), pp. 206-209.

[3] J. Liu, X. Cheng and J. Rao, "Development trend of operation simulator for port cranes", Hoisting and Conveying Machinery, vol. 39, no. 8, (2006), pp. 1-5.

[4] C. Wang, G. Liang and C. Liang, "The training simulator of container crane", Acta Simulata Systematica Sinica, vol. 14, no. 7, (2002), pp. 904-906.

[5] X. Jiang and W. Mi, "Design of chair's vibration prompting system based on training simulator", Chinese Journal of Construction Machinery, vol. 2, no. 3, (2004), pp. 319-323.

[6] B. Rong, X. Rui and G. Wang, "Developments of studies on multibody system dynamics", Journal of Vibration and Shock, vol. 30, no. 7, (2011), pp. 178-187.

[7] K. Li, Y. S. Qian and D. Xu, "A CA Model of Cultural Transmission and Its Dynamics Simulation”, JCIT: Journal of Convergence Information Technology, vol. 7, no. 15, (2012), pp. 90-98.

[8] T. Shiibaa and Y. Suda, "Development of driving simulator with full vehicle model of multibody dynamics", JSAE Review, vol. 6, no. 23, (2002), pp. 223-230.

[9] K. Lu, W. Mi, C. Chen and X. Wan, "Container Vehicle-Truss Bridge Coupled Vibration Analysis under Environmental Load for Automated Container Terminal", IJACT: International Journal of Advancements in Computing Technology, vol. 5, no. 4, (2013), pp. $499 \sim 506$. 
[10] Y. L. Xu, N. Zhang and H. Xia, "Vibration of coupled train and cable-stayed bridge system in cross wind", Bridge Engineering, ASCE, vol. 12, no. 26, (2004), pp. 1389-1306.

[11] X. Guo and Q. Zeng, "Analysis of critical wind speed for running trains on a schemed Yangtze River Bridge at Nanjing on Jing-Hu high speed railway line", Journal of The China Railway Society, vol. 23, no. 5, (2004), pp. 75-80.

[12] Y. Li, S. Qiang and H. Liao, “3-D coupled vibration of wind vehicle bridge system”, China Civil Engineering Journal, vol. 38, no. 7, (2005), pp. 61-64, 70.

\section{Author}

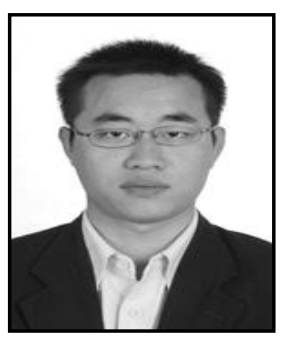

LU Kai-liang, received his $\mathrm{PhD}$ from Tongji University, Shanghai, China. Currently, he is working in Logistics Engineering College, Shanghai Maritime University (SMU) as a lecturer. His research interests include port machine structure and system dynamics, theory and method of dynamic design and optimization of structure, etc. He has 16 publications to his credit both in international and national Journals. $\mathrm{He}$ is a committee member of Shanghai Society of Theoretical \& Applied Mechanics, member of China Construction Machinery Society (CCMS), and Logistics Engineering Institution, CMES. 
International Journal of Hybrid Information Technology Vol.6, No.5 (2013) 